\title{
Validation of a Simplified, Portable Cardiopulmonary Gas Exchange System for Submaximal Exercise Testing
}

\author{
Andrew D. Miller ${ }^{1}$, Paul R. Woods ${ }^{1}$, Thomas P. Olson ${ }^{1}$, Minelle L. Hulsebus ${ }^{1}$, Kathy A. O’Malley ${ }^{1}$, \\ Dean MacCarter ${ }^{2}$ and Bruce D. Johnson ${ }^{*}, 1$
}

\author{
${ }^{I}$ Division of Cardiovascular Diseases, Department of Internal Medicine, Mayo Clinic, Rochester, MN, USA \\ ${ }^{2}$ Aurora Denver Cardiology Associates, Aurora, CO, USA
}

\begin{abstract}
Shape Medical Systems, Inc. has developed a new miniaturized, simplified system for non-invasive cardiopulmonary gas exchange quantification and has targeted their system for submaximal clinical exercise testing in order to abbreviate testing in an expanding clinical market during a climate of escalating health care costs. The focus of the present study was to compare this new device to a validated, standardized system for measures of cardiopulmonary gas exchange. Eighteen healthy adults (10 male $/ 8$ female, age $29 \pm 7 \mathrm{yr}$, BMI $23.8 \pm 2.4 \mathrm{~kg} / \mathrm{m}^{2}$ ) were brought to the laboratory and instrumented with both measurement systems via in-series pneumotachs. Additionally, the Shape system included a pulse oximeter for heart rate (HR) and oxygen saturation $\left(\mathrm{SaO}_{2}\right)$, while the standard system included separate 12-lead ECG and oximetry devices. The protocol included 2-min resting breathing, followed by 3-min at each of 3 workloads $(50,70,125$ watts) on a cycle ergometer. Data were collected breath-by-breath and averaged the last 30-sec of each workload. After a 15-min rest period, the pneumotach order was reversed and the study repeated. Since gas exchange data were similar $(p>0.05)$ within a given metabolic testing system between sessions the data were pooled for comparing the Shape and Standard systems. There were no differences $(\mathrm{p}>0.05)$ between the systems for oxygen consumption- $\mathrm{VO}_{2}$, carbon dioxide production $-\mathrm{VCO}_{2}$, ventilation- $\mathrm{V}_{\mathrm{E}}$, end tidal $\mathrm{CO}_{2}-\mathrm{PetCO}$, tidal volume- $\mathrm{V}_{\mathrm{T}}$, respiratory rate-fb, and $\mathrm{HR}$ at rest or any work load. $\mathrm{SaO}_{2}$ was slightly, but significantly lower using the Shape embedded oximeter ( $<<0.05$, averaging $\sim 1 \%$ across workloads). There were significant correlation coefficients $(\mathrm{p}<0.001)$ between the systems for $\mathrm{VO}_{2}(\mathrm{r}=0.991)$, $\mathrm{VCO}_{2}(\mathrm{r}=0.986), \mathrm{V}_{\mathrm{E}}(\mathrm{r}=0.995)$ and $\mathrm{PetCO}_{2}(\mathrm{r}=0.953)$. Bland Altman plots indicated good agreement between systems for the same gas exchange measures, independent of workload. The Shape system was sensitive to small increases in work (50 to 70 watts) and there were no differences in gas exchange measures in a subgroup of subjects ( $\mathrm{n}=4$ ) using a tight fitting mask instead of a mouthpiece $(\mathrm{p}>0.05)$. These data suggest that the new, simplified metabolic system developed by Shape Medical Systems, Inc. accurately quantifies key cardiopulmonary variables over a range of workloads, has a coefficient of variation similar to a well validated system and can be used with mouthpiece or mask.
\end{abstract}

Keywords: Oxygen consumption, primary care, metabolic, dyspnea.

\section{INTRODUCTION}

Cardiopulmonary exercise testing is playing an expanding role in the management of a variety of clinical populations, including heart failure, pulmonary hypertension, obstructive and restrictive lung disease [1]. Traditionally, non invasive measures of gas exchange have focused on determination of peak oxygen consumption $\left(\mathrm{VO}_{2 \text { peak }}\right)$, however, it is becoming increasingly clear that many submaximally determined gas exchange parameters (e.g., ventilatory efficiency $-\mathrm{V}_{\mathrm{E}} / \mathrm{VCO}_{2}$, end tidal $\mathrm{CO}_{2}-\mathrm{PetCO}_{2}$, oxygen efficiency slope) may be as important as peak variables for quantifying health status, determining prognosis, as well as following response to therapy [2-4]. There may also be advantages to clinical submaximal cardiopulmonary exercise testing including more reproducible measures, levels of activity that correspond to tasks of daily living, better patient compliance and reduced

*Address correspondence to this author at the Division of Cardiovascular Diseases, Gonda 5-369, Mayo Clinic, Rochester, MN 55905, USA; Tel: 507-284-4441; Fax: (507)266-7929; E-mail: johnson.bruce@mayo.edu potential for adverse events $[5,6]$. The testing environment and length of the test would also be greatly simplified, thus reducing test costs.

There are a number of automated metabolic measurement systems currently available on the market, each with specific strengths and limitations [7-11]. Typically however they require an integration of equipment, multiple test personnel and significant technical knowledge to run the systems and capture consistent and reliable data. Technical expertise is needed to perform relatively sophisticated calibration routines and some basic understanding of the algorithms used for assessment along with a skilled team for carrying out the testing (typically a nurse, exercise specialist and/or a physician) are needed. The instruments have tended to be bulky and require separate space for performing the testing along with code carts and other complimentary equipment (ECG machines) needed when exercising patients to their maximal capacity.

In view of these limitations various studies have examined simpler and more portable metabolic systems. However, the validity of these newer systems, e.g. the Jaeger 
Oxycon Mobile, remains controversial as systematic errors have been reported in gas exchange measurements [12].

More recently, a metabolic testing system has been developed (Shape Medical Systems, Inc, St. Paul, MN) that is small, portable, self calibrating (minimizing user error), with built-in automated temperature, barometric pressure, and humidity corrections. The user interface is simplified and software is designed so that extensive understanding of the measurements or equipment is not needed. The system relies on algorithms that were designed for light submaximal testing to obtain much of the same information gleaned from a maximal stress test. It relies on a simple 3-5 min, lightmoderate intensity exercise test to determine health status and can be performed in a doctor's office without need for complimentary monitoring equipment or sophisticated treadmills or cycle ergometers and can be used with a simple step test. The system however has not been validated with an established metabolic measurement system. Thus, the focus of this study was to compare gas exchange data obtained with this simplified system with a sophisticated, validated and calibrated state-of-the-art system currently used in our laboratory.

\section{MATERIALS AND METHODS}

\section{Participants}

Healthy active males and females (between the ages of 18 and $49 \mathrm{yr}$ ) were recruited from the surrounding area. Exclusion criteria included use of medications, history of cardiac or pulmonary abnormalities, obese (BMI $>27$ ), or orthopedic limitations. The study was reviewed and approved by the Mayo Clinic Institutional Review Board and all subjects reviewed and signed informed consent prior to participation.

\section{Overview of the Protocol}

Subjects reported to the laboratory and were simultaneously instrumented with the Shape simplified system and the "standard system". For the Shape system they were instrumented with a pulse oximeter (for heart rate) and a pneumotach attached in series with the standard system. For the standard system, subjects were instrumented with a 12-lead electrocardiogram (for heart rate and safety monitoring), a mouthpiece in series with pneumotachographs from both systems, along with a nose clip. Both systems were started simultaneously and data were collected in the following order, 2-min rest, 3-min 50 watts, 3-min 70 watts and 3-min 125 watts on a cycle ergometer. After a brief rest period (15 minutes) the order of the pneumotachs was reversed and the test repeated (see Fig. 1). The small increment from 50 to 70 watts provided a test for sensitivity of the systems to discriminate small changes in external work. Linking the pneumotachs in series allowed simultaneous data collection, however, this could have small influences on breath timing, phase relationships and dead space and thus the data were collected with the pneumotachs under both conditions for comparison.

\section{Standard System for Non invasive Gas Exchange Testing}

The standard system included a Medical Graphics CPX/D.(Medical Graphics, St Paul, MN) integrated to a Perkin Elmer Mass Spectrometer MGA 1100 (MA Tech
Services, Inc, St. Louis, MO) for gas analysis. This system using the Prevent Pneumotach has been previously validated using classical bag collection techniques and gravimetric quality gases for calibration of the mass spectrometer [13, 14]. In addition, characteristics of the system were optimized by testing individuals at multiple breathing rates and exercise loads $[13,15]$. Previously published data regarding this system resulted in a coefficient of variation for oxygen consumption- $\mathrm{VO}_{2}$ ranging from $8.7 \%$ at rest to $2.3 \%$ during heavy exercise [14]. An ECG was integrated with the Medical Graphics System to obtain heart rate on a breath by breath basis throughout testing.

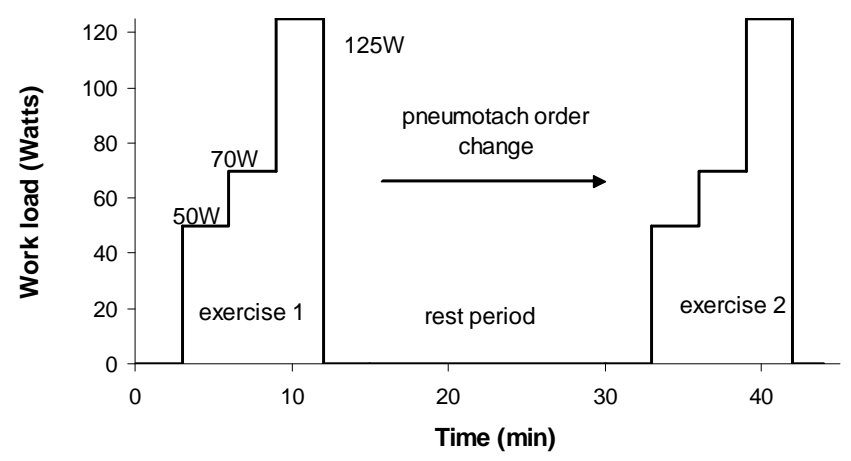

Fig. (1). Protocol for validating the Shape system for cardiopulmonary gas exchange measures. Subjects were instrumented with both systems by putting pneumotachs in series. Data were collected at rest, 50, 70 and 125 watts followed by a 15min rest period, reversing pneumotach order and repeating the testing.

\section{Shape System for Submaximal Cardiopulmonary Exercise Testing}

The Shape-HFTM Cardiopulmonary Exercise Testing System (St. Paul, MN) consisted of the following significant components, 1) Laptop Computer, 2) Shape-HF Data Analyzer, 3) Patient Interface, 4) Pulse Oximeter Finger Probe (Smiths Medical PM, Inc. 3711 BCI Digital Micro Power Board with w Comfort-Clip Oximetry Finger Sensor) (see Fig. 2). The laptop computer supplied with the ShapeHF system is OEM supplied operated with Windows $\mathrm{XP}{ }^{\circledR}$ operating software. Shape-HF system software is installed in the laptop computer.

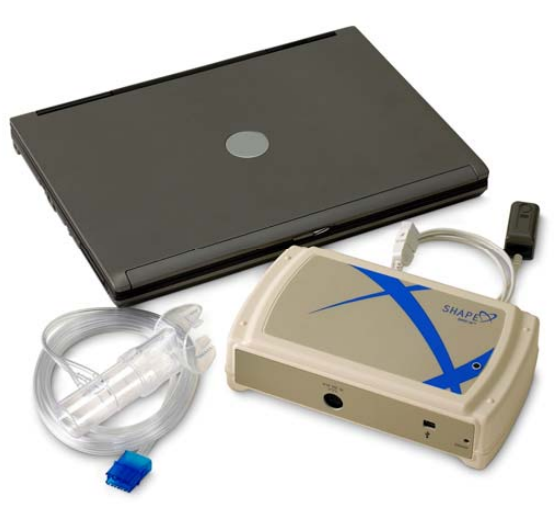

Fig. (2). The Shape system consists of a plug and play analyzer, lap top and pneumotach/sensor system. 
The Shape system is designed for use with a wide range of exercise testing modalities including simple stair stepping, cycle ergometry, treadmill, or other exercise modalities while the patient is connected to the Shape-HF Data Analyzer via the patient interface and pulse oximeter finger probe. The data analyzer contains discrete analyzers that measure expired oxygen and carbon dioxide, an airflow measurement subsystem that measures expired and inspired air using a fixed orifice, differential pressure pneumotach, and a pulse oximeter to measure heart rate and blood oxygen saturation. The data analyzer transmits measured data once per breath via a USB interface cable to the laptop computer. The system requires a laptop computer; however, future designs will allow standalone systems. Transmitted data includes breath count, PetCO $, \mathrm{VCO}_{2}, \mathrm{VO}_{2}$, tidal volume- $\mathrm{V}_{\mathrm{T}}$, breathing frequency-fb, heart rate-HR, barometric pressure, $\mathrm{SaO}_{2}$, dead space, peak expiratory flow, volume Inspired, resting energy, expiratory time, and inspiratory time. The patient interface consists of a fixed orifice, differential pressure pneumotach to which a respiratory gas sample line and upstream and downstream differential pressure sample lines are attached.

\section{Additional Testing}

A subset of subjects $(n=4)$ were brought back to the laboratory on a subsequent day to repeat testing using the Shape tight fitting mask. This was to assure that the use of a mask did not alter gas exchange data quality.

\section{Data Collection and Analysis}

Data were collected on a breath-by-breath basis using both systems with complete datasets for each subject downloaded into Excel. Data were then averaged over the last 30 -sec for each condition for each subject. Initial comparisons between exercise sessions for a given system did not reveal significant differences $(p>0.05)$ across measures and thus data from each session were averaged for further comparisons between the Shape and standard systems. Descriptive statistics (means, standard deviations and ranges) were computed with comparisons made between systems for $\mathrm{V}_{\mathrm{T}}, \mathrm{fb}, \mathrm{V}_{\mathrm{E}}, \mathrm{VO}_{2}, \mathrm{VCO}_{2}$, $\mathrm{HR}, \mathrm{SaO}_{2}$, and $\mathrm{PetCO}_{2}$ values using students t-tests $(\alpha=0.05)$. In addition, Pearson's correlation coefficients were calculated to determine linearity between systems and Bland-Altman plots were constructed to assess agreement between systems. Primary analyses for correlations and Bland-Altman plots focused on the measures of $\mathrm{VO}_{2}, \mathrm{VCO}_{2}, \mathrm{~V}_{\mathrm{E}}$ and $\mathrm{PetCO}_{2}$ as these measures take into account the integration of the primary measures used in cardiopulmonary gas exchange.

\section{RESULTS}

Subject characteristics are reported in Table 1. Subjects were young men and women with normal weight and blood pressure values.

The Shape system tracked closely with breath-by-breath changes measured by the standard system. Fig. (3) is an example of the breath-by-breath changes in $\mathrm{VO}_{2}$ in a subject transitioning from rest to the initial work loads (50 and 70 watts). Slight differences in phase are noted in part due to the sequence of pneumotachs and the ability to start the systems at identical time periods.
Table 1. Subject Characteristics $(n=18)$

\begin{tabular}{|l|c|c|}
\hline Age (yr) & $28.8 \pm 7.3$ & 18 to 46 \\
\hline Gender $\mathbf{( M / F )}$ & $(10 / 8)$ & - \\
\hline Ht $\mathbf{( c m )}$ & $173.4 \pm 11.4$ & 156.2 to 203.5 \\
\hline Wt $(\mathbf{k g})$ & $72.0 \pm 13.1$ & 54.0 to 93.2 \\
\hline BMI $\left(\mathbf{k g} / \mathbf{m}^{2}\right)$ & $23.8 \pm 2.4$ & 19.1 to 27.4 \\
\hline SBP $(\mathbf{m m H g})$ & $106.3 \pm 12.0$ & 94 to 136 \\
\hline DBP $(\mathbf{m m H g})$ & $66.6 \pm 9.4$ & 48 to 82 \\
\hline
\end{tabular}

$\mathrm{Ht}$, height; Wt, weight; BMI, body mass index; SBP, systolic blood pressure; DBP, diastolic blood pressure.

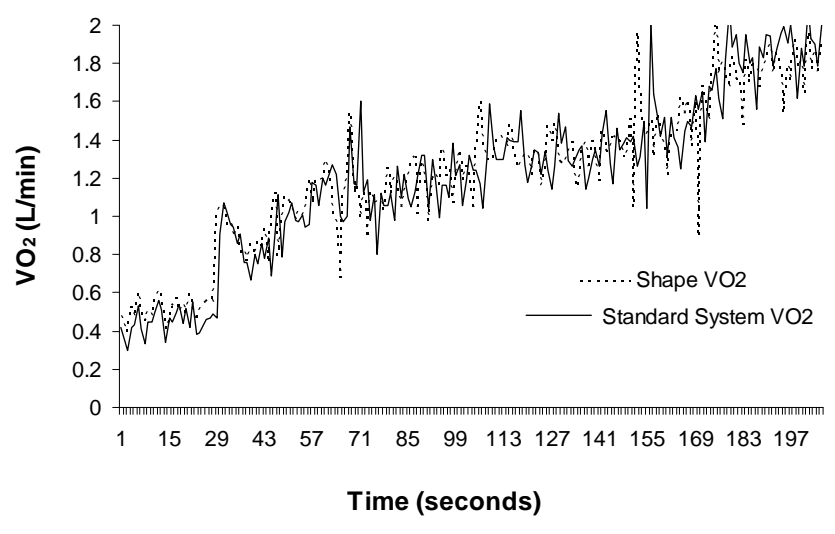

Fig. (3). Example of breath-by-breath plot of Shape and standard system for $\mathrm{VO}_{2}$ at rest and during the initial work intensities. Slight differences are noted in phase due to the differences in pneumotach order and slight variations in systems when attempting to start testing at identical time periods.

\section{Reproducibility of Shape Values Relative to the Standard System (Session 1 vs 2)}

The coefficient of variation $(\mathrm{CV})$ between sessions 1 and 2 for the Shape system ranged from 2.9 to $10.8 \%$ for $\mathrm{VO}_{2}$, 4.0 to $8.7 \%$ for $\mathrm{VCO}_{2}, 1.9$ to $3.3 \%$ for $\mathrm{V}_{\mathrm{E}}, 2.6$ to $9.3 \%$ for $\mathrm{HR}, 0.3$ to $0.4 \%$ for $\mathrm{SaO}_{2}$ and 2.9 to $4.3 \%$ for $\mathrm{PetCO}_{2}$. Typically the CV's were higher in resting data and reduced with exercise. The exception was HR, which was highest at 125 watts. The CV's for the standard system were similar to the Shape system during exercise, except for HR which ranged from 1.6 to $3.8 \%$. However this might be expected when comparing a 12-lead ECG to pulse oximetry. The CV's for $\mathrm{VO}_{2}$ and $\mathrm{VCO}_{2}$ at rest tended to be lower in the standard system at 4.1 and $5.4 \%$, respectively.

\section{Sensitivity of Shape System for Detecting Small Changes in Cardiopulmonary Measures}

In order to test the ability of the Shape system to accurately measure small changes in gas exchange variables, the protocol included a relatively small 20 watt increment in workload during the exercise session (50 $\rightarrow 70$ watts). Significant increases of similar magnitude were measured by both systems for $\mathrm{VO}_{2}(0.24 \pm 0.08$ vs $0.23 \pm 0.07 \mathrm{~L} / \mathrm{min}$, $\mathrm{p}<0.05), \mathrm{VCO}_{2}(0.27 \pm 0.08$ vs $0.28 \pm 0.07 \mathrm{~L} / \mathrm{min}, \mathrm{p}<0.05)$ and $\mathrm{V}_{\mathrm{E}}(5.8 \pm 2.4$ vs $6.3 \pm 2.3 \mathrm{~L} / \mathrm{min}$, for Shape and standard systems, respectively, $\mathrm{p}<0.05)$. 
Table 2. Gas Exchange/Heart Rate Data Comparing Shape System to Standard System

\begin{tabular}{|c|c|c|c|c|c|c|c|c|}
\hline $\begin{array}{c}\text { Variable } \\
\text { HR (bpm) }\end{array}$ & \multicolumn{2}{|c|}{ Rest } & \multicolumn{2}{|c|}{$50 \mathrm{~W}$} & \multicolumn{2}{|c|}{$70 \mathrm{~W}$} & \multicolumn{2}{|c|}{$125 \mathrm{~W}$} \\
\hline $\mathrm{fb}(\mathrm{bpm})$ & $12 \pm 4$ & $12 \pm 4$ & $21 \pm 6$ & $21 \pm 6$ & $23 \pm 5$ & $23 \pm 5$ & $26 \pm 6$ & $25 \pm 6$ \\
\hline $\mathrm{V}_{\mathrm{T}}(\mathrm{L})$ & $0.91 \pm 0.4$ & $0.98 \pm 0.41$ & $1.27 \pm 0.31$ & $1.31 \pm 0.36$ & $1.38 \pm 0.26$ & $1.47 \pm 0.29$ & $1.85 \pm 0.38$ & $2.00 \pm 0.42$ \\
\hline $\mathrm{VO}_{2}(\mathrm{~L} / \mathrm{min})$ & $0.26 \pm 0.12$ & $0.32 \pm 0.06$ & $1.00 \pm 0.18$ & $1.03 \pm 0.15$ & $1.24 \pm 0.1$ & $1.23 \pm 0.11$ & $1.80 \pm 0.2$ & $1.81 \pm 0.2$ \\
\hline $\mathrm{VCO}_{2}(\mathrm{~L} / \mathrm{min})$ & $0.22 \pm 0.11$ & $0.27 \pm 0.06$ & $0.79 \pm 0.13$ & $0.80 \pm 0.10$ & $1.04 \pm 0.11$ & $1.06 \pm 0.07$ & $1.67 \pm 0.17$ & $1.76 \pm 0.12$ \\
\hline $\mathrm{PetCO}_{2}(\mathrm{mmHg})$ & $34.5 \pm 3.4$ & $33.2 \pm 3.5$ & $39.2 \pm 2.1$ & $38.7 \pm 2.3$ & $40.4 \pm 2.3$ & $40.1 \pm 2.4$ & $41.8 \pm 3.4$ & $42.1 \pm 3.4$ \\
\hline $\mathrm{SaO}_{2}(\%)$ & $97.2 \pm 1.2$ & $98.1 \pm 1.8^{*}$ & $96.2 \pm 1.7$ & $97.9 \pm 1.2 *$ & $96.6 \pm 1.1$ & $97.8 \pm 1.2 *$ & $96.5 \pm 1.2$ & $97.7 \pm 1.3^{*}$ \\
\hline
\end{tabular}

$\mathrm{W}$, Watts; $\mathrm{HR}$, heart rate; fb, breathing frequency; $\mathrm{V}_{\mathrm{T}}$, tidal volume; $\mathrm{V}_{\mathrm{E}}$, minute ventilation; $\mathrm{VO}_{2}$, volume of oxygen consumed; $\mathrm{VCO}{ }_{2}$, volume of carbon dioxide consumed; PetCO partial pressure of end-tidal carbon dioxide; $\mathrm{SaO}_{2}$, arterial oxygen saturation. Data are reported as Mean \pm Standard Deviation. *p $<0.05$ between Shape and Standard.

\section{Comparison of Shape Cardiopulmonary Measures to the Standard System}

Gas exchange and HR data are provided in Table 2 for rest and each exercise stage. There were no significant differences between the mean data obtained from the Shape system $v s$ the mean values obtained from the standard system for the primary gas analysis measures $(p>0.05)$.

Bland-Altman plots indicated good agreement between the systems for $\mathrm{VO}_{2}, \mathrm{VCO}_{2}, \mathrm{~V}_{\mathrm{E}}$, and $\mathrm{PetCO}_{2}$. Plots are shown in Figs. $(\mathbf{4}, \mathbf{5})$ for rest and all workloads. The BlandAltman plots for $\mathrm{VO}_{2}$ demonstrated a mean for rest and all work levels of $0.180 \mathrm{~L} / \mathrm{min}$ with limits of agreement $\left(\mathrm{mean} \pm 2 \mathrm{SD}\right.$ ) of -0.178 to $0.228 \mathrm{~L} / \mathrm{min}$. $\mathrm{VCO}_{2}$ demonstrated a mean of $0.043 \mathrm{~L} / \mathrm{min}$ with limits of agreement of -0.178 to $0.260 \mathrm{~L} / \mathrm{min}$. $\mathrm{V}_{\mathrm{E}}$ demonstrated a mean of $1.13 \mathrm{~L} / \mathrm{min}$ with limits of agreement of -1.31 to $3.56 \mathrm{~L} / \mathrm{min}$. PetCO demonstrated a mean of -0.56 with limits of agreement of 2.40 to $1.52 \mathrm{mmHg}$. These findings coincide with excellent correlation coefficients between Shape and the standard metabolic measurement system (not shown) for $\mathrm{VO}_{2}$ $(\mathrm{r}=0.991, \mathrm{P}<0.0001$, Root Mean Square Error [RMSE] $=$ 0.076), $\mathrm{VCO}_{2}(\mathrm{r}=0.986, \mathrm{P}<0.001, \mathrm{RMSE}=0.089), \mathrm{V}_{\mathrm{E}}$ $(\mathrm{r}=0.995, \mathrm{PV} 0.001, \mathrm{RMSE}=1.601)$ and $\mathrm{PetCO}_{2}(\mathrm{r}=0.953$, $\mathrm{P}<0.001$, RMSE $=1.341)$.

\section{Shape Mask vs Mouthpiece}

In a subgroup of subjects we compared a tight fitting mask to the mouthpiece using the Shape system only. Correlation coefficients ranged from 0.94 for $\mathrm{PetCO}_{2}$ to 0.98 for $\mathrm{VO}_{2}, \mathrm{VCO}_{2}$ and $\mathrm{V}_{\mathrm{E}}(\mathrm{p}<0.001)$ with no significant difference between values at rest or any of the work intensities $(p>0.05)$. This suggests that the mask is a valid tool for use with the Shape system when measuring cardiopulmonary exercise variables.

\section{DISCUSSION}

\section{Overview of Main Findings}

There is a need for simplified measurement systems to assess cardiopulmonary gas exchange in the clinical setting. The purpose of this study was to test a new simplified, miniaturized device developed by Shape Medical Systems,
Inc. which is specifically designed for 'plug and play' submaximal exercise testing. This system compared favorably to our previously validated "state-of-the-art" cardiopulmonary gas exchange system that integrates the Medical Graphics CPX/D metabolic cart with a Perkin Elmer mass spectrometer and a GE 12-lead electrocardiographic system. The Shape system tracked small changes in external work and no systematic differences were observed across the primary cardiopulmonary gas exchange variables used in clinical exercise testing. In addition, the Shape system had similar CV's for repeat testing compared to the standard system during exercise. Moreover, the use of a tight fitting mask provided similar gas exchange measures when compared to those made using a mouthpiece.

The protocol used allowed for the system pneumotachs to be aligned in series which facilitated simultaneous quantification of dynamic breath-by-breath changes in gas exchange during exercise. In addition, to obviate any issues due to slight differences in dead space or phase relationships when one system was down stream from the other, we tested subjects twice after reversing the pneumotach order. We performed submaximal testing (average HR $<140 \mathrm{bpm}$ and RER $<1.0$ at 125 watts) and thus there was little metabolic carry over into the second session. In fact, cardiopulmonary measures did not significantly differ from session 1 to 2 which allowed for the pooling of data for comparisons between systems. The strong, highly significant correlation coefficients are consistent with minimal differences between the two systems.

\section{Benefits of Submaximal Cardiopulmonary Exercise Testing}

The use of non invasive cardiopulmonary gas exchange testing has expanded into a number of clinical areas and is now used frequently to characterize disease status, determine response to therapy and gain perspective on prognosis $[1,3$, 16-20]. Limitations to further expansion of this testing to other clinical areas include space requirements, adequately trained personnel, the complexity and time for testing as well as the significant costs. In contrast, the Shape system has simplified procedures by eliminating physical calibration routines, miniaturizing the device, simplifying the computer interface and focusing on submaximal low level exercise 
$\mathrm{VO}_{2}$

Rest

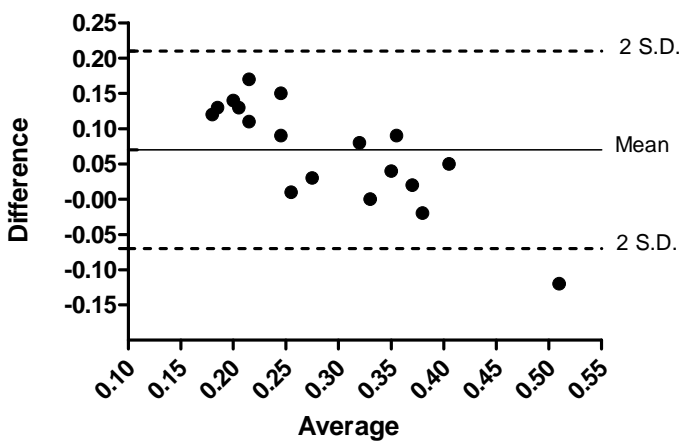

$50 \mathrm{~W}$

$70 \mathrm{~W}$
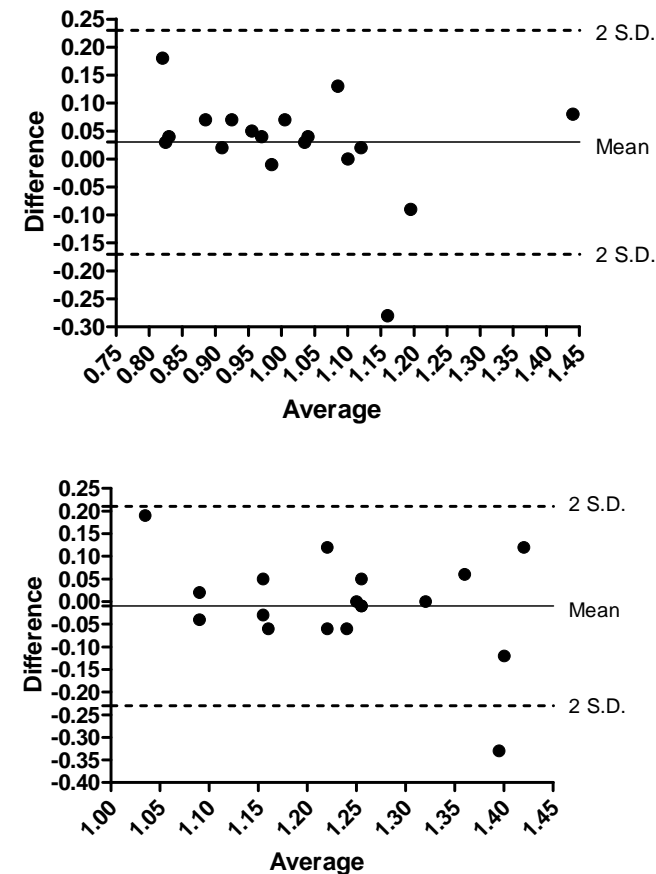

$125 \mathrm{~W}$

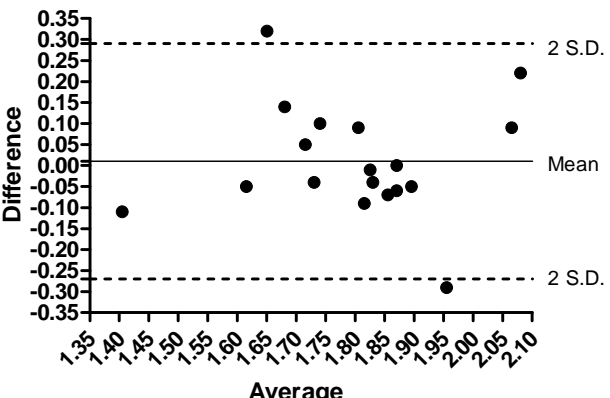

Fig. (4). Bland-Altman plots for $\mathrm{VO}_{2}$ and $\mathrm{VCO}_{2}$ (standard - Shape).

testing, thus meeting a potential market need. The system operates by using a vacuum to pull in gas samples from a patient's inhalation and exhalation which is then split and sent to an $\mathrm{O}_{2}$ and $\mathrm{CO}_{2}$ sensor and analyzed simultaneously. The $\mathrm{O}_{2}$ cell is a standard chemical cell, used in various products, while the $\mathrm{CO}_{2}$ cell is a calibrated and temperature compensated infrared sensor. Flow rates are determined
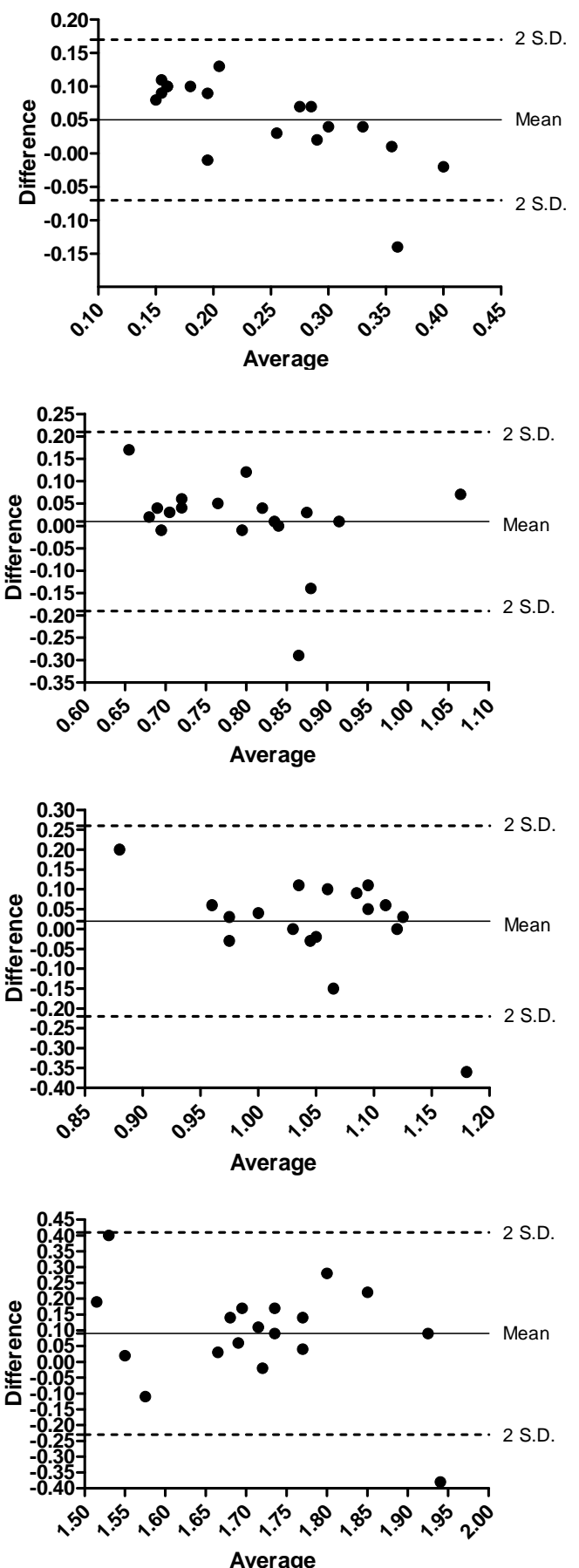

using traditional differential pressure measurements through a fixed orifice plate.

A number of studies have examined submaximal measures of cardiopulmonary testing. Measures such as ventilatory efficiency slope, $\mathrm{O}_{2}$ efficiency slope, $\mathrm{PetCO}_{2}$ and HR recovery slope all appear to be good prognostic indicators; in many cases as good as peak values [21]. In 


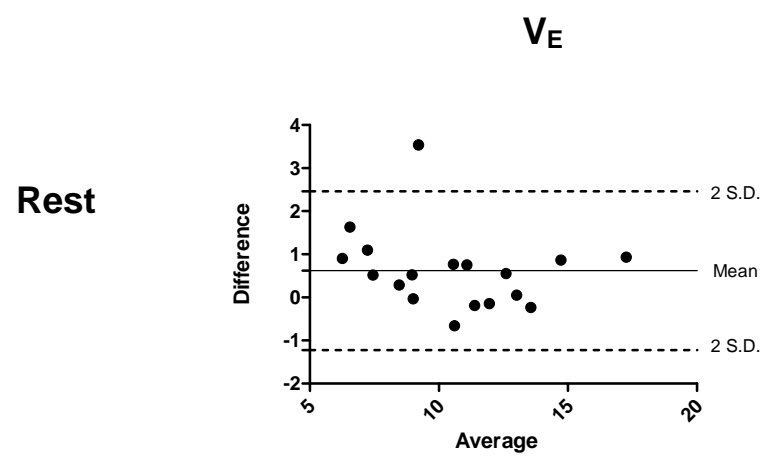

\section{$\mathrm{PetCO}_{2}$}

$50 \mathrm{~W}$

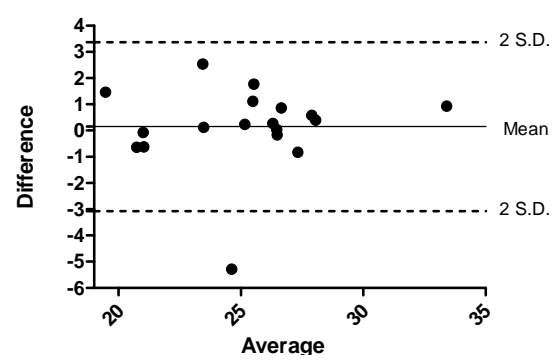

$70 \mathrm{~W}$

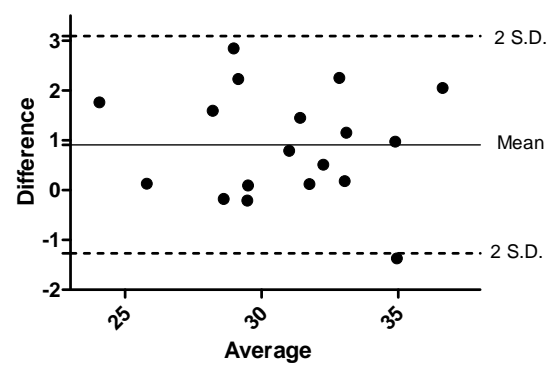

$125 \mathrm{~W}$

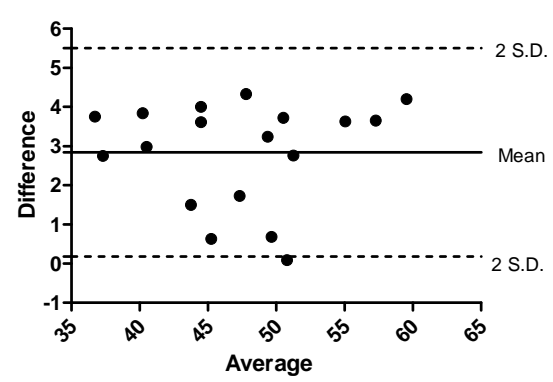

Fig. (5). Bland-Altman plots for $\mathrm{V}_{\mathrm{E}}$ and $\mathrm{Pet} \mathrm{CO}_{2}$ (standard - Shape).

addition, many of these measures may be more reproducible than maximal values since they are not effort dependent $[5$, $6,22]$.

\section{Validity of the Standard System for Comparison}

The system used as the "gold" standard for which to compare the Shape system was the Medical Graphics CPX/D integrated with mass spectrometry. This system has been validated against classical bag collection techniques and further optimized using various breathing patterns [13]. In addition, this system is maintained using strict quality control standards including routine physiologic calibrations in addition to the specific calibration procedures conducted prior to each testing session. The integrated mass spectrometer has clearly been shown to be the most stable

system for gas analysis; able to measure several gases simultaneously, quickly and without the complication of water vapor. This automated system was chosen as the standard to validate the Shape system in order to be able to track breath-by-breath changes and to optimize collection timing. In addition, there are other short comings to the bag collection method. Lucia and colleagues report, among other concerns [10], the cumbersome inability to make simultaneous real time sample collections as a primary limitation of the bag collection method. Additionally, others have suggested that the ideal experimental design when validating metabolic measurement systems would have simultaneous breath-by-breath data collection [11]. The CPX/D has been successfully used as a reference system during rest and varying intensities of exercise elsewhere [7, 9, 22, 23]. 
Collectively, the CPX/D metabolic measurement system is a valid and reliable metabolic measurement system.

\section{LIMITATIONS}

The Shape system is dependent on pulse oximetry for heart rate and does not contain a 12-lead ECG for detecting silent ischemia or arrhythmias. As demonstrated, the $\mathrm{CV}$ for heat rate ranged from $3.8 \%$ at rest to $2.4 \%$ at 50 and $2.6 \%$ at 70 watts, but increased to $9.3 \%$ at the 125 watt workload. Excessive hand movement or gripping the cycle ergometer may have played a role in the variability, though subjects were generally asked to keep a loose grip on the handle bars. The mean HR did not differ between systems and the CV was within generally acceptable ranges. Other oximetry probes (e.g., forehead) have the potential to reduce this variability [25].

The Shape system is also limited by the orifice restrictor plate in the pneumotach and hence the amount of volume that can flow through it. The Pneumotach can theoretically handle flow rates up to $300 \mathrm{~L} /$ minute; however, we have not tested it beyond the values reported in this manuscript. The reason for this is that the device in general is designed for low level submaximal exercise testing which is the clinical impetus for the product to be used quickly and safely in a physician office.

\section{CONCLUSIONS}

The system developed by Shape Medical System, Inc tracks changes in key cardiopulmonary gas exchange measures accurately up to modest levels of exertion and provides a novel, simplified system for office based clinical cardiopulmonary exercise testing.

\section{ACKNOWLEDGEMENTS}

The authors would like to thank the participants.

\section{REFERENCES}

[1] Bettencourt P, Ferreira A, Dias P, et al. Predictors of prognosis in patients with stable mild to moderate heart failure. J Card Fail 2002; 6: 306-13.

[2] Arena R, Brubaker P, Moore B, Kitzman D. The oxygen uptake efficiency slope is reduced in older patients with heart failure and a normal ejection fraction. Int J Cardiol 2009. [Epub ahead of print]

[3] Arena R, Guazzi M, Myers J. Prognostic value of end-tidal carbon dioxide during exercise testing in heart failure. Int J Cardiol 2007; 117: 103-8.

[4] Arena R, Tevald M, Peberdy MA, Turner T. Prognostic value of phase 1 of oxygen uptake on-kinetics response in the heart failure population: a pilot study. J Cardiopulmon Rehabil 2004; 24: 401-4.

[5] Davies LC, Wensel R, Georgiadou P, et al. Enhanced prognostic value from cardiopulmonary exercise testing in chronic heart failure by non-linear analysis: oxygen uptake efficiency slope. Eur Heart J 2006; 27: 684-90.
[6] Hollenberg M Tager IB. Oxygen uptake efficiency slope: an index of exercise performance and cardiopulmonary reserve requiring only submaximal exercise. J Am Coll Cardiol 2000; 36: 194-201.

[7] Byard AD, Dengel DR. Validity of a portable metabolic measurement system. Med Sci Sports Exerc 2002; 34: S149.

[8] Corporation MG. The MedGraphics VO2000 User Guide. St. Paul, MN (Part number 142121-001 Rev. A). 2000.

[9] Hausswirth C, Bigard AX, Le Chevalier JM. The Cosmed K4 telemetry system as an accurate device for oxygen uptake measurements during exercise. Int J Sports Med 1997; 18: 449-53.

[10] Lucia A, Fleck SJ, Gotshall RW, Kearney JT. Validity and reliability of the Cosmed K2 instrument. Int J Sports Med 1993; 14: 380-6.

[11] Macfarlane DJ. Automated metabolic gas analysis systems: a review. Sports Med 2001; 31: 841-61.

[12] Diaz V, Benito PJ, Peinado AB, et al. Validation of a new portable metabolic system during an incremental running test. J Sports Sci Med 2008; 7: 532-6.

[13] Proctor DN, Beck KC. Delay time adjustments to minimize errors in breath-by-breath measurement of $\mathrm{VO}_{2}$ during exercise. J Appl Physiol 1996; 81: 2495-9.

[14] Johnson BD, Beck KC, Proctor DN, Miller J, Dietz NM, Joyner MJ. Cardiac output during exercise by the open circuit acetylene washin method: comparison with direct Fick. J Appl Physiol 2000; 88: 1650-8.

[15] Beck KC, Gore CJ. Optimizing breath-by-breath $\mathrm{VO}_{2}$ measurements. Med Sci Sports Exerc 2004; 36: 554-5.

[16] Abraham MR, Olson LJ, Joyner MJ, Turner T, Beck KC, Johnson $\mathrm{BD}$. Angiotensin-converting enzyme genotype modulates pulmonary function and exercise capacity in treated patients with congestive stable heart failure. Circulation 2002; 106: 1794-9.

[17] Agostoni P, Pellegrino R, Conca C, Rodarte JR, Brusasco. Exercise hyperpnea in chronic heart failure: relationships to lung stiffness and expiratory flow limitation. J Appl Physiol 2002; 92: 1409-16.

[18] Arena R, Myers J, Abella J, et al. The prognostic value of the heart rate response during exercise and recovery in patients with heart failure: Influence of beta-blockade. Int J Cardiol 2010; 138: 166173.

[19] Chase P, Arena R, Myers J, et al. Relation of the prognostic value of ventilatory efficiency to body mass index in patients with heart failure. Am J Cardiol 2008; 101: 348-52.

[20] Guazzi M, Myers J, Peberdy MA, Bensimhon D, Chase P, Arena R. Ventilatory efficiency and dyspnea on exertion improvements are related to reduced pulmonary pressure in heart failure patients receiving Sildenafil. Int J Cardiol 2009 [Epub ahead of print].

[21] Robbins M, Francis G, Pashkow FJ , et al. Ventilatory and heart rate responses to exercise: better predictors of heart failure mortality than peak oxygen consumption. Circulation 1999; 100: 2411-7.

[22] Guazzi M, Reina G, Tumminello G, Guazzi MD. Exercise ventilation inefficiency and cardiovascular mortality in heart failure: the critical independent prognostic value of the arterial $\mathrm{CO} 2$ partial pressure. Eur Heart J 2005; 26: 472-80.

[23] Schulz H, Helle S, Heck H. The validity of the telemetric system CORTEX X1 in the ventilatory and gas exchange measurement during exercise. Int J Sports Med 1997; 18: 454-7.

[24] Olson TP, Tracy JE, Dengel DR. Validity of a low-flow pneumotach and portable metabolic system for measurement of basal metabolic rate. Med Sci Sports Exerc 2003; 35: S143.

[25] Tokuda K, Hayamizu K, Ogawa K, Hirai T, Irita K. A comparison of finger, ear and forehead $\mathrm{SpO} 2$ on detecting oxygen desaturation in healthy volunteers. Anesthesiology 2007; 107: A1544. 